\title{
Repeated Spatial Acquisition: Effects of NMDA Antagonists and Morphine
}

\author{
Mark Galizio, Julian R. Keith, Will J. Mansfield, and Raymond C. Pitts \\ University of North Carolina at Wilmington
}

\begin{abstract}
Effects of morphine and $2 \mathrm{~N}$-methyl-D-aspartate (NMDA) receptor antagonists, phencyclidine and LY235959, were studied using a within-subject, repeated-acquisition/performance procedure adapted to the Morris Swim Task. In the performance component, subjects swam to a hidden platform that was always in the same location in the pool. In the acquisition component, the platform was moved to a different place for each session. Baseline training produced rapid and direct swims to the platform in the performance component and steep within-session learning curves in the acquisition component. All 3 compounds increased swim distances, escape latencies, and slowed swim speed in a dose-dependent manner, but only morphine consistently produced selective impairments on acquisition. NMDA antagonists generally affected acquisition only at doses that also disrupted performance, although phencyclidine produced selective effects in some animals. These outcomes were different than those from studies of response chains in primates, suggesting that task and species variables may be important determinants of drug effects on acquisition.
\end{abstract}

There is considerable interest in the therapeutic potential of $N$-methyl-D-aspartate (NMDA) receptor antagonists because of their neuroprotective, anticonvulsant, anti-Parkinsonian, and anxiolytic actions, as well as their ability to attenuate the development of tolerance and expression of dependence to opioids (Allen \& Dykstra, 2000a, 2000b; Bisaga et al., 2001; Parsons, Danysz, \& Quack, 1999; Wiley, 1997). Although high doses of NMDA antagonists are contraindicated for most purposes because they produce gross behavioral impairments such as hallucinations, ataxia, and stereotypy, lower doses may provide therapeutic benefits without untoward side effects. There has, however, been controversy with respect to the effects of lower doses (CorySlechta, 1994; Willetts, Balster, \& Leander, 1990). For example, there has been considerable interest in the possibility that NMDA antagonists may produce learning and memory impairments at doses that do not disrupt more general performance processes.

Mark Galizio, Julian R. Keith, Will J. Mansfield, and Raymond C. Pitts, Department of Psychology, University of North Carolina at Wilmington.

The University of North Carolina at Wilmington Animal Care and Use Committee approved these procedures. This research was supported by a grant from the National Institute on Drug Abuse (DA12879). We thank Heather Bell, Daryl Buda, Amanda McGann, Patrick McKinney, Laurence Miller, Diane Nelson, Diana Padlubnaya, and Elise Redmond for their help with behavioral testing. We also thank David Leander, Lilly Laboratories, for the generous gift of LY235959 and the National Institute on Drug Abuse for the morphine, naloxone, and phencyclidine used in this study.

Correspondence concerning this article should be addressed to Mark Galizio, Department of Psychology, University of North Carolina at Wilmington, Wilmington, North Carolina 28403-5612. E-mail: galizio@uncw.edu
Much of the research addressing the cognitive effects of NMDA antagonists has focused on spatial learning by rats in the Morris swim task (MST; Morris, 1981). In this procedure the rat is placed into a large circular pool at various randomly determined start locations and swims to a submerged escape platform located in a fixed position with respect to extrapool stimuli. After a few trials, escape latencies become shorter and the rat's path to the platform more direct, and these indices are taken to indicate spatial learning. Several studies have reported that competitive (e.g., DL-2-amino-5-phosphopentanoic acid [AP5]) and noncompetitive (e.g., dizocilpine [MK801]) NMDA antagonists impair acquisition of navigation in the MST at doses that also block hippocampal long-term potentiation (e.g., Heale \& Harley, 1990; Morris, Anderson, Lynch, \& Baudry, 1986; Robinson, Crooks, Shinkman, \& Gallagher, 1989). Impaired learning occurred at doses that did not affect performance of a control group on a cued task (e.g., one in which the platform is visible) in these studies, so it appeared that the effects could not be accounted for by sensory, motor, or other less specific processes (see McNamara \& Skelton, 1993).

However, a series of studies by Cain et al. (Cain, Ighanian, \& Boon, 2000; Cain, Saucier, \& Boon, 1997; Saucier, Hargreaves, Boon, Vanderwolf, \& Cain, 1996) demonstrated that the spatial learning impairments caused by NMDA antagonists were eliminated when the investigators provided procedural pretraining (teaching subjects to swim away from the pool walls and to climb onto the escape platform) in one environment before testing drug effects on learning to swim to a hidden platform in a different setting. Cain et al. concluded that the effects of NMDA antagonists in naive rats were not specific to learning processes but rather were likely due to more general sensorimotor impairments. 
The studies reviewed previously on the effects of NMDA antagonists on spatial learning used research designs that made indirect comparisons between groups of subjects (i.e., pretrained vs. naive subjects; hidden vs. visible escape platform groups) and usually examined the effects of only a single drug dose. However, to address the difficulties of separating the behavioral effects of drugs that directly involve learning processes from those that simply affect the performance of a learned task, more sophisticated techniques may be necessary. One important example is the multiple-component repeated-acquisition/performance procedure, which involves training patterns of operant responding, usually on chain or tandem schedules (Thompson \& Moerschbaecher, 1979). In one component of a multiple schedule (performance component), a single specific response sequence is always reinforced throughout the experiment; however, in the presence of the stimuli in the other (acquisition) component, the reinforced response sequence changes each session. Drug effects are investigated only after acquisition and performance baselines are established and stable. Although these kinds of procedures require more extensive training to establish stable behavioral baselines before drug testing, they offer several advantages over other procedures. For example, they permit the evaluation of complete dose-effect functions from individual animals and allow direct, within-session comparisons of drug effects on the acquisition of a novel sequence of behavior with the performance of one previously learned (see Cohn \& Paule, 1995; Thompson \& Moerschbaecher, 1979).

Several studies have examined noncompetitive NMDA antagonists using repeated-acquisition procedures in monkeys. For example, Moerschbaecher, Thompson, and Winsauer (1985) found that phencyclidine (PCP) interfered with the acquisition of a new response sequence at doses that did not affect performance of a well-trained sequence, and similar selective effects on acquisition were reported for PCP and ketamine by Thompson, Winsauer, and Mastropaolo (1987). Using procedures that compared acquisition and performance of conditional discriminations, Moerschbaecher et al. also found that dizocilpine and PCP produced selective impairment of acquisition (France, Moerschbaecher, \& Woods, 1991; Moerschbaecher \& Thompson, 1980).

Fewer multiple-component repeated-acquisitions/performance studies are available with rats as subjects, perhaps because of the difficulties associated with developing the complex baseline behaviors required. Baron and Moerschbaecher (1996) found that both dizocilpine and the competitive NMDA antagonist CGS 19755 impaired acquisition of a response sequence in one group of rats at doses that did not impair performance of a well-learned sequence in a different group. However, using a within-subject design with rats, Cohn and Cory-Slechta (1993) found only very slight effects of dizocilpine on acquisition until doses that also affected performance were reached.

Although some studies have used repeated-acquisition procedures in the study of spatial learning (Brooks, CorySlechta, Murg, \& Fedoroff, 2000; Peele \& Baron, 1988; Whishaw, 1985), Keith and Galizio (1997) adapted the multiple-component repeated-acquisition/performance procedure to the swimming pool navigation task. Keith and Galizio investigated the effects of dizocilpine (MK-801) on spatial learning and performance. Rats were trained in two different swimming pools and learned to swim to a hidden escape platform that was always in the same location in one pool (performance component) and moved to a different place for each daily session in a second pool (acquisition component), alternating between the two components within each session. Consistent baseline behavior rapidly developed in which rats learned the platform location in the acquisition component within the first few trials of the session (i.e., rapid decreases in within-session escape latencies) while maintaining stable baseline escape latencies throughout the performance component. Dizocilpine impaired acquisition in a dose-dependent manner, but doses that increased acquisition escape latencies $(\geq 0.2 \mathrm{mg} / \mathrm{kg})$ also produced clear evidence of impairment in the performance component. These results were consistent with conclusions reached by Cain et al. (e.g., Saucier et al., 1996) that effects of NMDA antagonists in the swim task are relatively nonspecific, but they seem at odds with studies of repeated acquisition of behavioral sequences and conditional discriminations in which effects selective to acquisition have generally been observed (e.g., France et al., 1991; Moerschbaecher et al., 1985). One purpose of the current study was to provide a systematic replication of Keith and Galizio (1997) with a noncompetitive NMDA antagonist (PCP) and a competitive antagonist (LY235959).

There are also intriguing inconsistencies between spatial learning and repeated acquisition in nonspatial (e.g., lever press) procedures in the literature with opiate compounds. For example, McNamara and Skelton $(1991,1992)$ found that morphine disrupts acquisition of navigation in the swim task. However, drug effects on more general performance could not be ruled out in these studies. In contrast, studies of morphine and other $\mu$-agonists using nonspatial, repeatedacquisition procedures have fairly consistently failed to report effects on acquisition except at doses that also disrupted performance in monkeys. For example, $\mu$-agonists typically produce nonselective effects on repeated acquisition of conditional discriminations (Moerschbaecher \& Thompson, 1983) and of response sequences (Moerschbaecher et al., 1985). Opiates have not been studied in rats using multiple-component repeated-acquisition/performance procedures, rendering it unclear whether the discrepancies in the findings from different studies reflect species differences, differences between spatial and nonspatial tasks, or other factors. Thus, a second purpose of the current study was to attempt to clarify this situation by using the Keith and Galizio (1997) procedure to determine the effects of morphine on repeated spatial acquisition in rats.

\section{Method}

\section{Subjects}

Subjects were 12 Holtzman Sprague-Dawley male rats. They were between 90 and 150 days old at the start of testing and were 
housed individually under continuous dim illumination with continuous access to food and water.

\section{Apparatus}

The apparatus was a circular white fiberglass pool $(1.5 \mathrm{~m}$ diameter, $30.5 \mathrm{~cm}$ deep) filled with $23 \mathrm{~cm}$ of water. A cylindrical platform made of white plastic (10 cm diameter, $20 \mathrm{~cm}$ high) was submerged such that the lip was $2 \mathrm{~cm}$ below the surface of the water. White nontoxic paint powder was used to make the water opaque. The water temperature was maintained at $30{ }^{\circ} \mathrm{C}\left( \pm 2{ }^{\circ} \mathrm{C}\right)$.
Plastic shower curtains enclosed the pool, and these formed the distinct stimulus configurations used to signal component changes and provide distal cues during navigation training (Figure 1). Two halogen torchlights $(500 \mathrm{~W})$ located outside the curtains illuminated the pool by reflection off the white painted ceiling. Subjects' movements within the pool were tracked and recorded using a video-tracking system that included a digital video camera mounted directly above the center of the pool attached to a microcomputer running data acquisition software (Polytrack, San Diego Instruments, San Diego, CA). This system permitted recording of escape latencies, the path traversed, and the total distance of
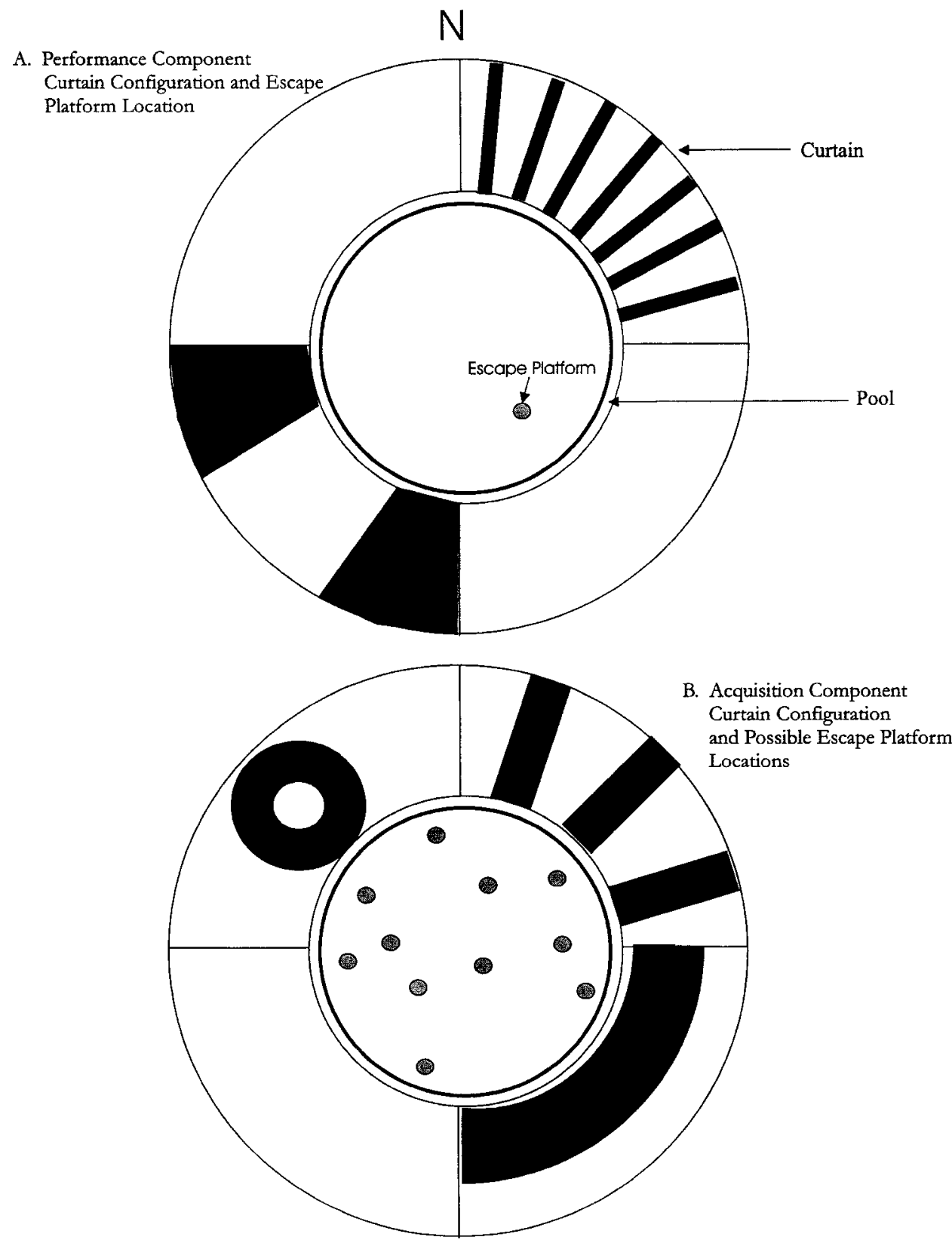

Figure 1. The stimulus arrangement present during training under the performance (A) and acquisition (B) components. Visual patterns were displayed on a curtain that encircled the apparatus. 
the path. A video monitor displayed an overhead view of the pool. Nontoxic black dye was used to mark the fur of each rat for video-tracking purposes.

\section{Procedure}

Preliminary training. Each rat received six trials per day with the escape platform in a fixed location. To begin a trial, a subject was gently placed into the water facing the wall of the pool at one of four starting points (North, South, East, or West determined randomly). If the rat failed to climb onto the platform in $60 \mathrm{~s}$, it was placed on the platform by the experimenter. Otherwise, when the rat reached the platform it remained there for $15 \mathrm{~s}$. Rats were then returned to their home cages for a 2.5-min intertrial interval (ITI). Preliminary training continued until subjects met a criterion of three consecutive sessions with escape latencies for each session averaging less than $10 \mathrm{~s}$ per trial. The curtain configuration and escape platform location used throughout preliminary training (and subsequently to define the performance component) is shown in the top panel of Figure 1.

Multiple-component training. During this phase, subjects received six training trials per session on each of two components distinguished by unique curtain patterns. In the performance component, the submerged platform remained at the same location throughout the course of the experiment. For each subject, the performance task occurred in the presence of the curtain configuration used for that subject during preliminary training. Responding in the acquisition component occurred in the presence of a different configuration of curtain patterns (see bottom panel of
Figure 1), and the submerged platform was always moved to a location different from that used in the preceding session. The platform locations used during the acquisition component are shown in Figure 1 (recall that the platform position during training on the acquisition component remained constant during individual sessions but changed each session). The sequence of platform positions was randomly determined with the constraint that the same position was not used on consecutive days. The sequence of release points used was also randomly determined with the constraint that the rat was not released from the same point on consecutive trials.

For each subject, training trials alternated between the performance and acquisition components. Each session began with a trial in the performance component and was followed by a 2.5-min ITI and then a trial in the acquisition component. Subjects received six trials in each component in each daily session (sessions were conducted 5 days a week, Monday through Friday) until they met a 10-session stability criterion. Mean latency for the most recent five sessions was subtracted from the mean latency of the immediately preceding five sessions, and the difference could not exceed $15 \%$ of the 10 -session mean (cf. Perone, 1991). This criterion was applied to both the performance and acquisition components. In addition, subjects had to average less than $10 \mathrm{~s}$ per trial in the performance task for all six trials and under $10 \mathrm{~s}$ per trial in the acquisition task for Trials 2 to 6 during the final 10 sessions. The stability and performance criteria had to be met simultaneously before the drug administration phase of the experiment began. Table 1 shows the number of sessions required to meet criteria for each subject.

Table 1

Number of Sessions Required to Reach Training Criteria and Number of Drug Dose Determinations for Each Rat

\begin{tabular}{|c|c|c|c|c|c|c|c|c|c|}
\hline Rat & Pretrain & Baseline & Saline & $0.3 \mathrm{mg} / \mathrm{kg}$ & $1 \mathrm{mg} / \mathrm{kg}$ & $3 \mathrm{mg} / \mathrm{kg}$ & $5.6 \mathrm{mg} / \mathrm{kg}$ & $10 \mathrm{mg} / \mathrm{kg}$ & Morphine + naloxone \\
\hline \multicolumn{10}{|c|}{ Morphine study } \\
\hline S6 & 8 & 15 & 4 & & 4 & 4 & & 4 & 2 \\
\hline S8 & 13 & 13 & 4 & & 4 & 4 & & 3 & 2 \\
\hline S9 & 5 & 22 & 4 & & 4 & 4 & & 3 & 2 \\
\hline S11 & 8 & 21 & 2 & & 2 & 2 & & 2 & 2 \\
\hline H10 & 7 & 22 & 4 & & 3 & 3 & & 3 & \\
\hline M11 & 8 & 20 & 3 & & 3 & 3 & & 3 & \\
\hline
\end{tabular}

Naloxone study

\begin{tabular}{lrrrrrrr} 
S6 & 8 & 15 & 3 & 2 & 3 & 3 & 3 \\
S8 & 13 & 13 & 3 & 2 & 3 & 3 & 3 \\
S9 & 5 & 22 & 3 & 2 & 3 & 3 & 3 \\
S11 & 8 & 21 & 2 & & 2 & 2 & 2 \\
\hline
\end{tabular}

LY235959 study

\begin{tabular}{lrrrrrr} 
A92 & 11 & 11 & 5 & 3 & 2 & 2 \\
A95 & 7 & 13 & 4 & 2 & 3 & 3 \\
A98 & 5 & 19 & 3 & 2 & 3 & 3 \\
M16 & 3 & 17 & 4 & 3 & 3 & 3 \\
M22 & 10 & 42 & 5 & 3 & 5 & 4 \\
M23 & 6 & 22 & 3 & 4 & 3 & 2 \\
\hline
\end{tabular}

$\begin{array}{lrrrrrrr}\text { A92 } & 11 & 11 & 2 & 2 & 3 & 3 & 3 \\ \text { A95 } & 7 & 13 & 3 & 2 & 2 & 3 & 4 \\ \text { A98 } & 5 & 19 & 3 & 2 & 4 & 4 & 3 \\ \text { M22 } & 10 & 42 & 3 & 2 & 3 & 3 & 1 \\ \text { I1 } & 4 & 16 & 3 & 3 & 2 & 4 & 2 \\ \text { I3 } & 9 & 35 & 3 & 3 & 4 & 4 & 2\end{array}$




\section{Drug Preparation and Administration}

Drug solutions were prepared on each test day by dissolving each compound in an isotonic $(0.9 \%)$ sodium chloride solution. Drug and saline injections (intraperitoneal) were administered in a volume of $1 \mathrm{ml} / \mathrm{kg}$ twice per week (Tuesday and Friday) $15 \mathrm{~min}$ before behavioral testing began. Dose-effect functions were designed to test a range of doses from one that produced no effect to one that substantially disrupted swimming. Six rats were tested with morphine (National Institute on Drug Abuse [NIDA]) in doses of $1.0,3.0$, and $10.0 \mathrm{mg} / \mathrm{kg}$ (all doses are expressed in terms of the total salt). After completion of the morphine study, 4 of these rats were also studied after receiving combinations involving injections of $3.0 \mathrm{mg} / \mathrm{kg}$ morphine (or saline) followed $5 \mathrm{~min}$ later by $1.0 \mathrm{mg} / \mathrm{kg}$ naloxone (or saline); for 3 of these rats, a dose-effect function was established for naloxone alone (NIDA; 0.3, 1.0, 3.0, and $10.0 \mathrm{mg} / \mathrm{kg}$ ). The other 8 rats were studied under PCP (NIDA; $0.3,1.0,3.0$, and $5.6 \mathrm{mg} / \mathrm{kg}[5.6 \mathrm{mg} / \mathrm{kg}$ was added because most rats did not show overall performance disruption at $3.0 \mathrm{mg} / \mathrm{kg}]$ ) or LY235959 (Lilly Pharmaceuticals, Indianapolis, IN, 0.3, 1.0, 3.0 $\mathrm{mg} / \mathrm{kg}$ ) or a combination. Four of these rats received both PCP and LY235959 and the other 4 received only one drug, resulting in 6 rats studied in each condition. For those subjects studied under both drugs, at least 10 baseline sessions were completed before the second drug study began. Drug doses were administered in an irregular order with the constraints that no dose was administered on successive drug days and that the end of each cycle (one exposure to each dose, including a saline injection) of the drug regimen was completed before beginning the next cycle. Each dose was determined two to three times except when substantial variability in reaction to the initial determinations was observed, in which case additional determinations were studied to obtain a more representative result. Table 1 shows the number of determinations of each drug dose for each subject.

\section{Dependent Variables}

One key measure was escape latency, or the time from placement in the pool until the rat's forepaws made contact with the escape platform. Escape latency is determined by the accuracy of the rat's path to the platform as well as overall swimming speed. Thus, a second measure of interest was an estimate of navigation accuracy: the swim path ratio. The path ratio reflects the difference between the actual swimming distance and the minimum possible distance from the release location to the submerged escape platform. It was computed using the following formula: (AD $\mathrm{MD} / \mathrm{MD}$, where $\mathrm{MD}$ is the minimal distance and $\mathrm{AD}$ is the actual distance swum on a given trial. A path ratio of zero would indicate that the subject swam the minimum possible distance in route to the escape platform, whereas higher ratios indicate less direct routes. Finally, actual swim speed was computed using distance and latency information automatically recorded by the tracking system for each trial.

\section{Data Analysis}

In addition to data from sessions preceded by saline injection, control data points from baseline sessions not preceded by an injection are provided. Separate Dose $\times$ Component factorial repeated measures analyses of variance (ANOVAs) were performed on each dependent variable. The mean of all determinations of each dose was entered into the analysis for each rat (baseline data were not included). When the ANOVAs revealed statistically significant effects, post hoc comparisons (Tukey's honestly significant difference tests) were computed to determine whether effects at particular doses differed reliably from those obtained during saline control sessions. Individual subject data are presented to permit more detailed analysis of drug effects.

\section{Results}

All rats showed rapid learning during the initial training, which involved swimming to a platform in a fixed position,
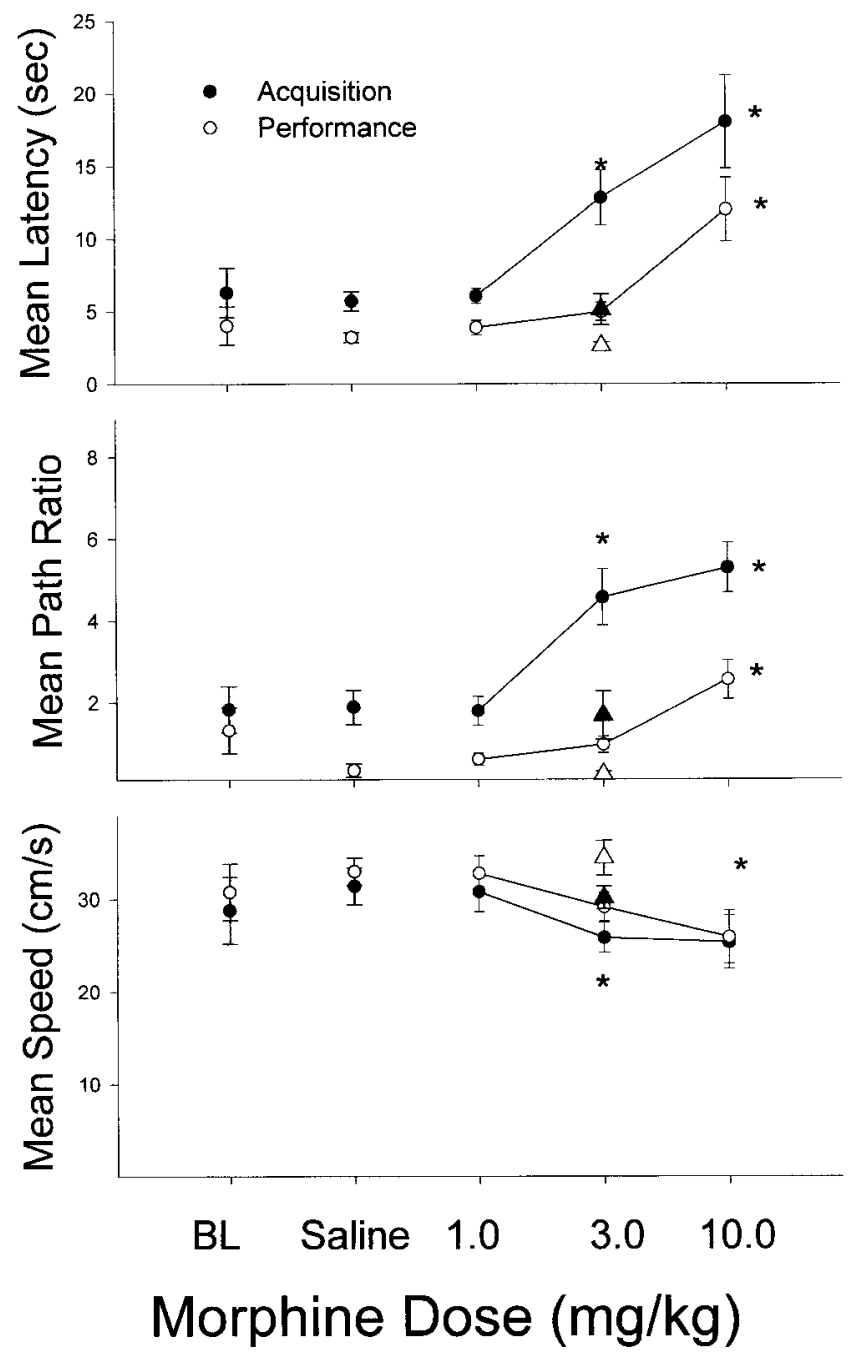

Figure 2. Mean escape latencies (top), swim path ratios (middle), and swim speeds (bottom) as a function of morphine dose during acquisition and performance components. Because acquisition was generally evident after the first trial, Trials 2-6 were used to compute acquisition means. All six trials were used to compute performance means. Means for each subject were computed for all determinations of each dose used to compute the group mean presented here. Bars indicate standard error of the mean. When error bars are not visible, they are smaller than the symbol used to represent the data point. Points that differ significantly from saline controls are indicated by asterisks $(p<.05)$. Triangles represent means obtained when an injection of $1.0 \mathrm{mg} / \mathrm{kg}$ of naloxone followed the morphine injection. $\mathrm{BL}=$ baseline, or means of sessions not preceded by an injection; sec $=$ seconds. 
Table 2

Mean Escape Latency as a Function of Naloxone Dose for Individual Rats

\begin{tabular}{|c|c|c|c|c|c|c|c|c|c|c|}
\hline \multirow[b]{2}{*}{ Rat } & \multicolumn{5}{|c|}{ Acquisition } & \multicolumn{5}{|c|}{ Performance } \\
\hline & Saline & $0.3 \mathrm{mg} / \mathrm{kg}$ & $1 \mathrm{mg} / \mathrm{kg}$ & $3 \mathrm{mg} / \mathrm{kg}$ & $10 \mathrm{mg} / \mathrm{kg}$ & Saline & $0.3 \mathrm{mg} / \mathrm{kg}$ & $1 \mathrm{mg} / \mathrm{kg}$ & $3 \mathrm{mg} / \mathrm{kg}$ & $10 \mathrm{mg} / \mathrm{kg}$ \\
\hline S6 & 5.67 & 8.16 & 5.17 & 5.20 & 6.37 & 2.57 & 3.54 & 3.16 & 4.29 & 2.87 \\
\hline S8 & 4.90 & 4.91 & 5.08 & 4.37 & 3.85 & 4.49 & 5.53 & 5.96 & 3.08 & 5.36 \\
\hline S9 & 5.21 & 7.42 & 4.82 & 3.39 & 4.84 & 3.14 & 3.61 & 2.93 & 2.18 & 2.10 \\
\hline S11 & 4.36 & - & 6.68 & 6.41 & 5.04 & 3.00 & - & 2.57 & 2.90 & 6.82 \\
\hline$M$ & 5.04 & - & 5.43 & 4.84 & 5.03 & 3.30 & - & 3.65 & 3.11 & 4.28 \\
\hline
\end{tabular}

meeting criterion in a mean of 7.4 sessions (range $=4-13$ sessions). More extended training was needed to meet the stability and performance criteria on the multiple-component task $($ mean $=20.6$ sessions, range: $11-42$ sessions; see Table 1). However, once subjects met these criteria, consistent patterns of baseline behavior were generally maintained throughout the subsequent drug regimen.

Figure 2 shows the effects of morphine on escape latency, path ratio, and swim speed. Morphine reliably affected swimming in a dose-dependent fashion across all dependent measures. Increases in latencies and path ratios and de- creases in swim speed as a function of morphine dose were confirmed by statistically significant main effects of dose for latency, $F(3,15)=18.24, p<.05$, path ratio, $F(3$, $15)=27.59, p<.05$, and speed, $F(3,15)=19.68, p<.05$. However, Figure 2 reveals that the effects of morphine on latency and path ratio occurred at lower doses in the acquisition component. At $3.0 \mathrm{mg} / \mathrm{kg}$, latencies and ratios were increased in the acquisition but not the performance component. This selective effect on acquisition was confirmed by significant Dose $\times$ Component interactions for latency, $F(3,15)=4.49, p<.05$, and path ratio, $F(3,15)=3.99$,

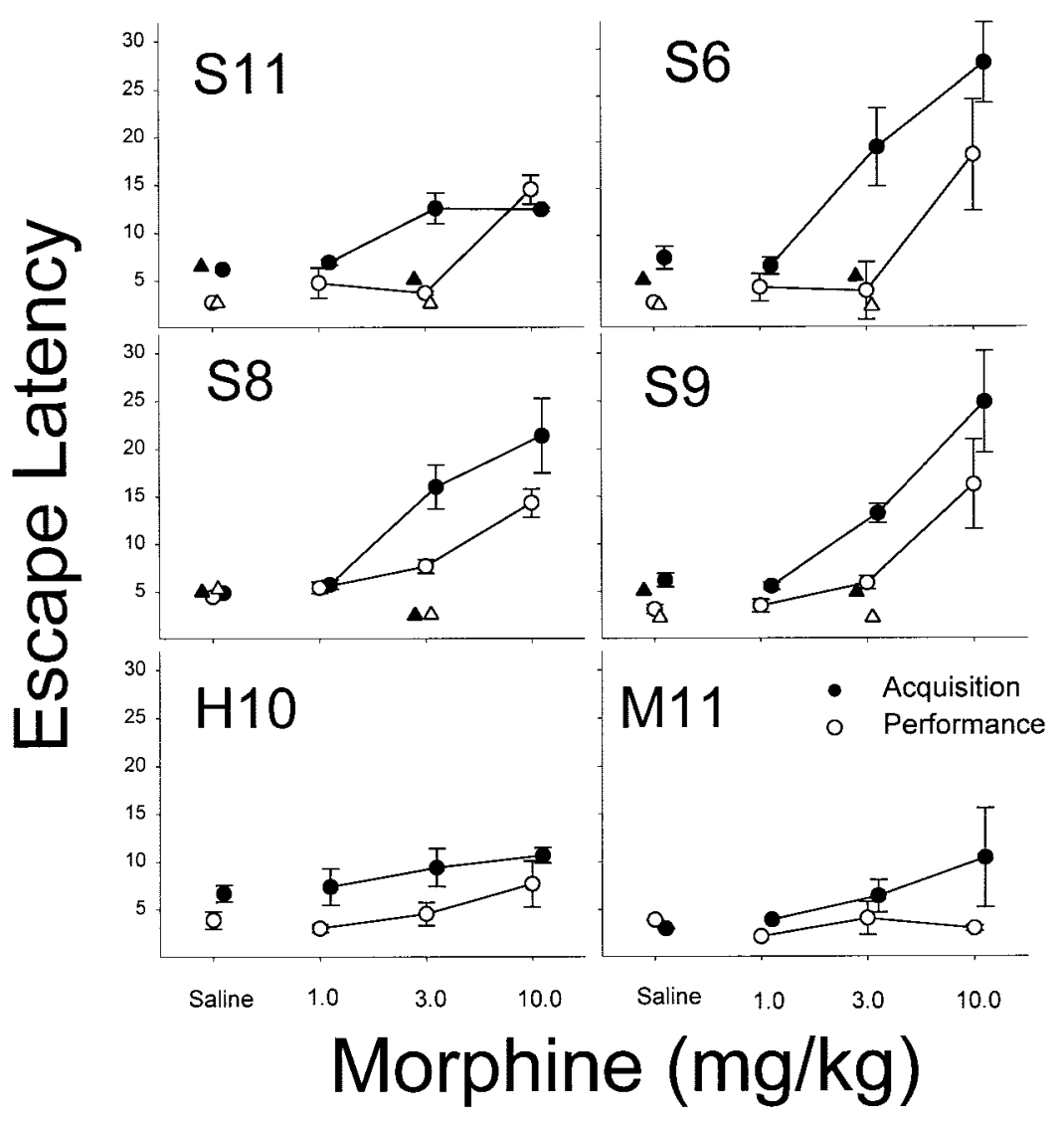

Figure 3. Individual escape latencies are presented as a function of morphine dose during acquisition and performance. Each point represents the means of all determinations of each dose, and vertical lines indicate standard error. Acquisition component means are based on Trials 2-6 of the session. Performance component means are based on all six trials. Triangles represent means obtained when an injection of $1.0 \mathrm{mg} / \mathrm{kg}$ of naloxone followed the morphine injection. 


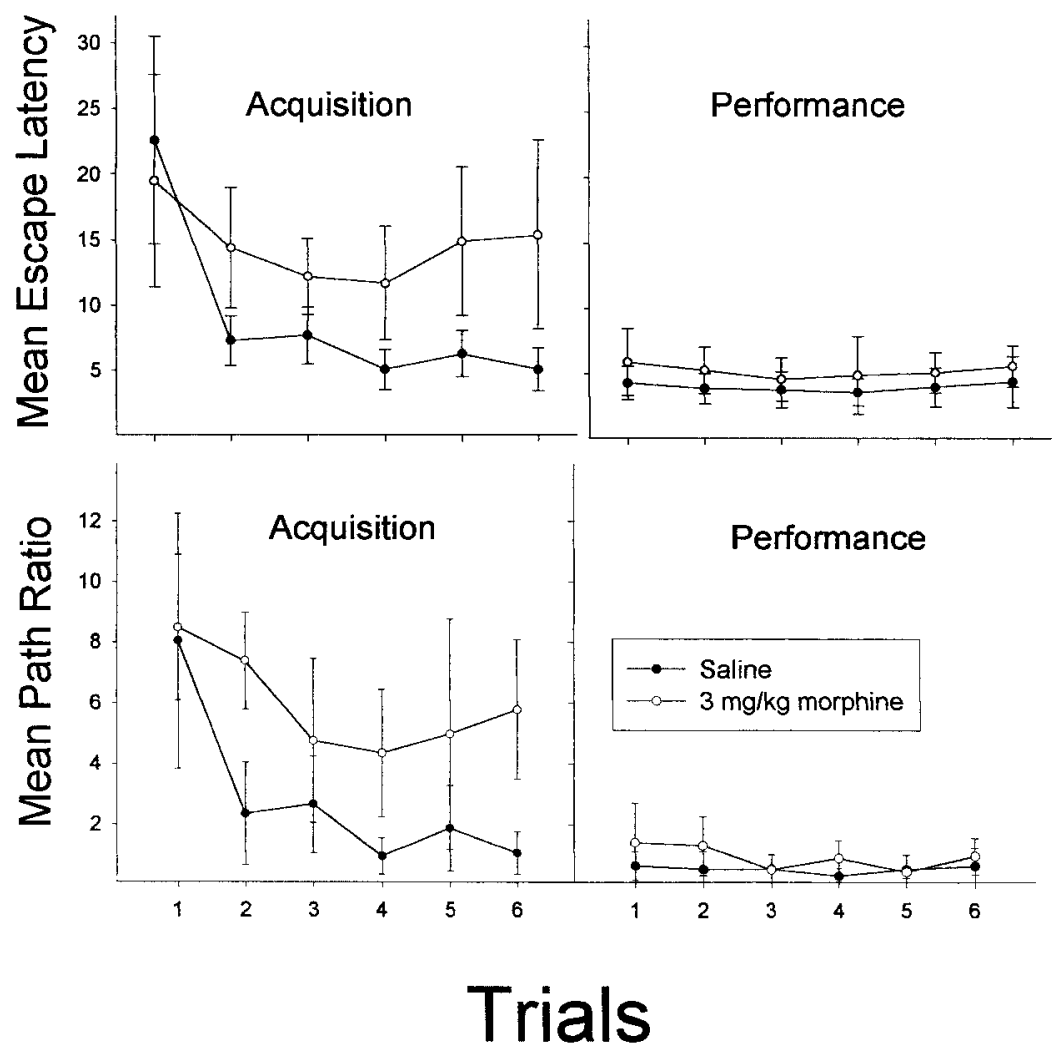

Figure 4. Mean escape latencies (top) and swim path ratios (bottom) during performance and acquisition components are presented as a function of trial within session for $3.0 \mathrm{mg} / \mathrm{kg}$ morphine (the dose that produced selective acquisition impairment) compared with baseline (saline). Bars indicate standard error of the mean. When error bars are not visible, they are smaller than the symbol used to represent the data point.

$p<.05$. These were based on reliable post hoc differences between the $3.0 \mathrm{mg} / \mathrm{kg}$ dose and saline in the acquisition $(p<.05)$ but not the performance component for both measures. The Dose $\times$ Component interaction for swim speed was nonsignificant, but post hoc tests indicated that the overall decreased speeds at both 3.0 and $10.0 \mathrm{mg} / \mathrm{kg}$ doses differed significantly from baseline $(p<.05)$.

Figure 2 also shows the results of sessions preceded by combinations of $3.0 \mathrm{mg} / \mathrm{kg}$ morphine and $1.0 \mathrm{mg} / \mathrm{kg}$ naloxone. The effects of morphine on latency and path ratio in the acquisition component were reversed by naloxone, and the decreases in swim speed noted in both components at 3.0 $\mathrm{mg} / \mathrm{kg}$ morphine also returned to baseline levels when that dose was administered in combination with naloxone. Naloxone dose-effect data for escape latency for the rats that completed this phase of the experiment are presented in Table 2. None of the naloxone doses resulted in statistically significant differences from baseline conditions on any measure.

Figure 3 presents acquisition and performance latency data from individual subjects. Five of the six rats showed clear selective effects of morphine. Four rats (S6, S8, S9, and S11) showed striking increases in latency at $3.0 \mathrm{mg} / \mathrm{kg}$ in the acquisition component with little or no effect in the performance component. Another rat (M11) also showed this effect but only at the $10 \mathrm{mg} / \mathrm{kg}$ dose of morphine. Thus, M11 also showed selective morphine effects but appeared to be less sensitive.

Figure 4 shows a trial-by-trial analysis of mean escape latencies and mean path ratios during acquisition and performance, specifically a comparison of $3 \mathrm{mg} / \mathrm{kg}$, the dose most frequently associated with selective effects, and saline. The saline data nicely illustrate typical baseline performances with long latencies and high ratios on Trial 1 of the acquisition component and rapid reduction over trials such that by Trial 4 both escape latencies and path ratios declined to the same low levels seen across all trials in the performance component. In contrast, acquisition latencies and ratios remained elevated even through Trial 6 after $3 \mathrm{mg} / \mathrm{kg}$ morphine, but neither measure was affected in the performance component.

Figure 5 shows the effects of LY235959 on escape latency, path ratio, and swim speed. LY235959 did not affect any of the measures until the $3.0 \mathrm{mg} / \mathrm{kg}$ dose was reached, at which point increases in latencies and path ratios and decreases in swim speed were observed in both components. These effects were confirmed by statistically significant main effects of dose for latency, $F(3,15)=21.12, p<.05$, 

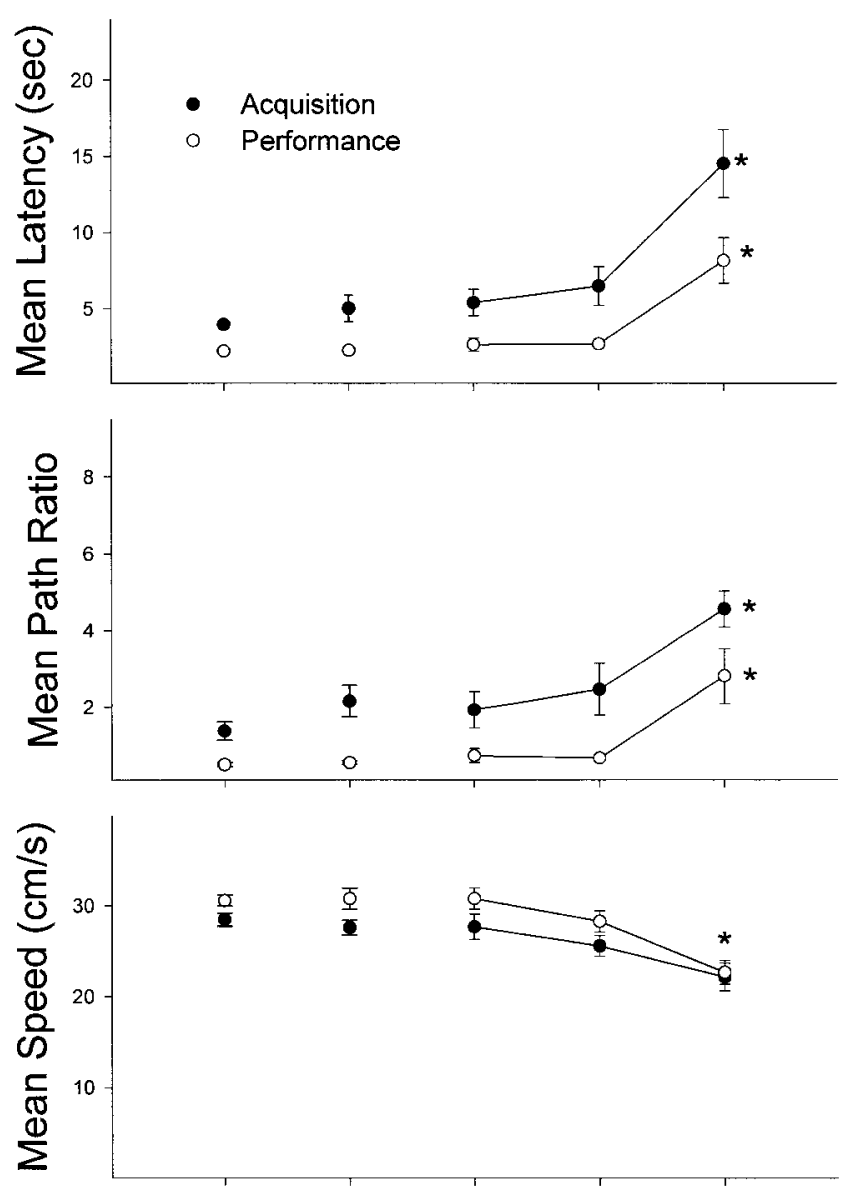

\section{BL Saline $\quad 0.3 \quad 1.0 \quad 3.0$ LY235959 (mg/kg)}

Figure 5. Mean escape latencies (top), swim path ratios (middle), and swim speeds (bottom) as a function of LY235959 dose during acquisition and performance components. Means for each subject were computed for all determinations of each dose used to compute the group mean presented here. When error bars are not visible, they are smaller than the symbol used to represent the data point. Points that differ significantly from saline controls are indicated by asterisks $(p<.05)$. BL $=$ baseline, or means of sessions not preceded by an injection; sec $=$ seconds.

path ratio, $F(3,15)=25.92, p<.05$, and speed, $F(3$, $15)=15.55, p<.05$, and post hoc tests revealed that the effects were significant only at the $3.0 \mathrm{mg} / \mathrm{kg}$ dose. The absence of significant Dose $\times$ Component interactions for latency, $F(3,15)=1.29, p>.05$, and path ratio $(F<1)$ is consistent with the parallel increases apparent in Figure 5 for both measures in the acquisition and performance components. Individual latency data, shown in Figure 6, generally were consistent with the conclusions based on group means. (Note, however, the elevated latency shown by Rat $\mathrm{M} 22$ at the $1.0 \mathrm{mg} / \mathrm{kg}$ dose in acquisition.)

PCP impaired swimming in a dose-dependent fashion across all dependent measures (Figure 7). Increases in la- tencies and path ratios and decreases in swim speed as a function of PCP dose were confirmed by statistically significant main effects of dose for latency, $F(4,20)=12.75$, $p<.05$, path ratio, $F(4,20)=13.46, p<.05$, and speed, $F(4,20)=18.53, p<.05$. Although parallel effects on acquisition and performance were apparent at the 5.6 dose for all the measures $(p<.05)$, it appears that $3.0 \mathrm{mg} / \mathrm{kg}$ PCP affected acquisition but not performance with respect to latency and path ratio. However, the Dose $\times$ Component interaction needed to support a claim for selective effects across subjects was not significant for either latency, $F(4$, $20)=1.45, p>.05$, or path ratio, $F(4,20)=1.89, p>.05$. The basis for the discrepancy between the trends observed in Figure 7 and the statistical analyses is apparent on inspection of the individual subject data (Figure 8). Latencies were strongly affected in acquisition, but not performance, for 2 rats at the $3.0 \mathrm{mg} / \mathrm{kg}$ dose. The selective effects in these 2 rats elevated the group means but were not consistently observed among subjects and thus not sufficient to yield a statistically significant outcome in the group analysis. Interestingly, however, at the $5.6 \mathrm{mg} / \mathrm{kg}$ dose Rat A92 showed a sharp increase in latency that was confined to the acquisition component. Thus, 3 of the 6 rats showed selective effects on acquisition at some dose of PCP, although the particular dose that produced selective effects was not the same for each.

\section{Discussion}

Morphine consistently increased escape latency and path ratio in the acquisition components at doses $(3.0 \mathrm{mg} / \mathrm{kg}$ in 4 rats and $10.0 \mathrm{mg} / \mathrm{kg}$ in a fifth) that were not accompanied by impairments on these measures in the performance components of the task. Under control conditions in the acquisition component, learning was rapid, as indicated by sharply reduced escape latencies after just one or two trials. However, after morphine, acquisition was markedly delayed, and escape latency never reached control levels (see Figure 4). In contrast, the competitive NMDA antagonist LY235959 generally impaired acquisition only at the highest dose (3.0 $\mathrm{mg} / \mathrm{kg}$ ), which also impaired performance. PCP, a noncompetitive NMDA antagonist, was more likely to produce selective effects than LY235959; 3 of 6 rats showed selective effects under PCP at some dose, but the effects were inconsistent in that the other 3 rats' acquisition was impaired only at doses that impaired performance.

The selective morphine effects are interesting for several reasons. Although other studies have found that morphine interfered with acquisition in the swim task, it was considered likely that these effects were due to a more generalized swimming impairment (McNamara \& Skelton, 1991, 1992). In the current study, overall swimming speed did slow significantly both at the 3.0 and $10.0 \mathrm{mg} / \mathrm{kg}$ doses of morphine, but impaired swimming does not seem sufficient to account for the selective decreases in accuracy in the acquisition component at the $3.0 \mathrm{mg} / \mathrm{kg}$ morphine dose (as indicated by the increased path ratio in acquisition and absence of an effect on path ratio in performance). 


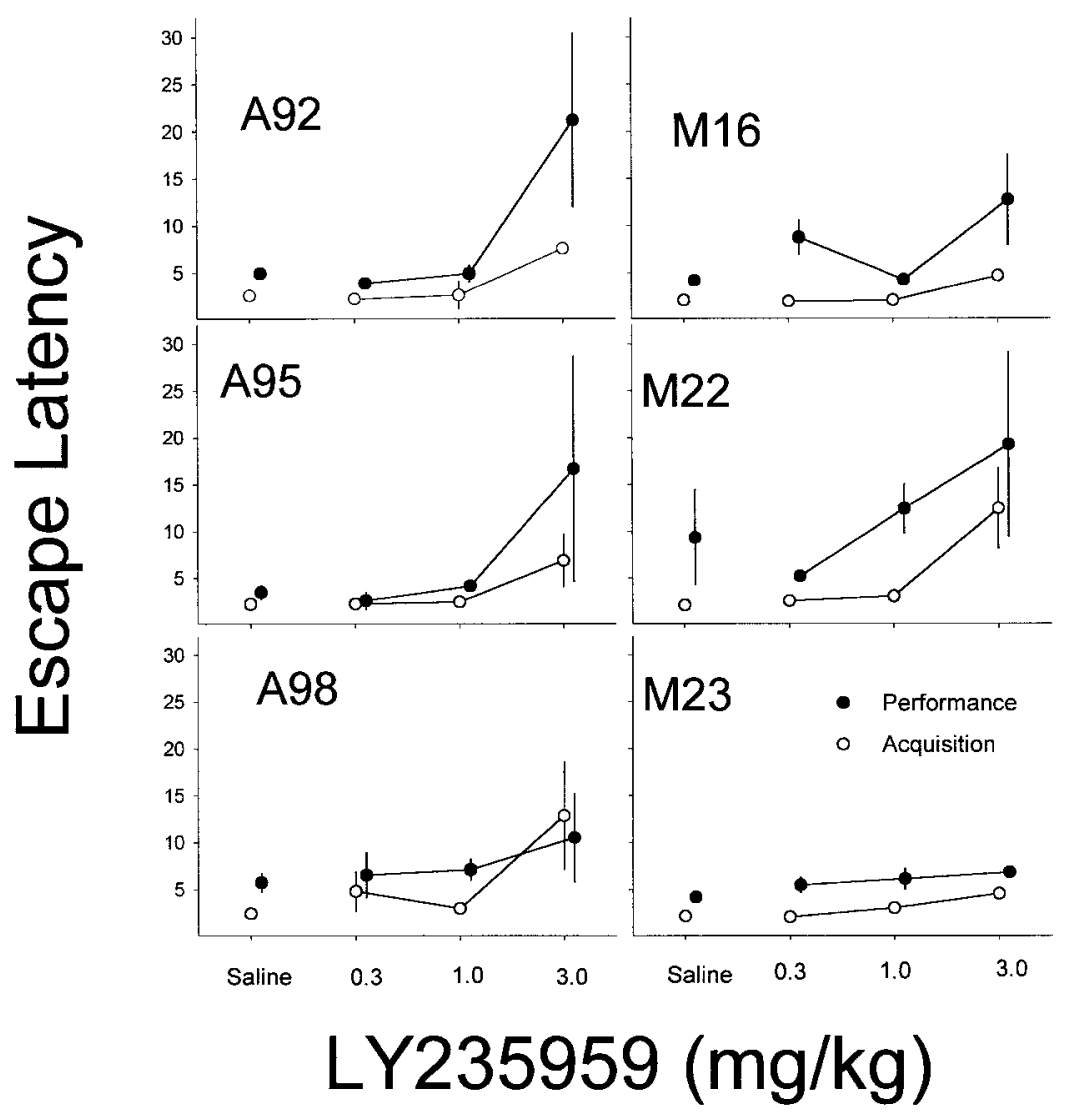

Figure 6. Individual escape latencies are presented as a function of LY235959 dose during acquisition and performance. Each point represents the means of all determinations of each dose, and vertical lines indicate standard error. Acquisition component means are based on Trials 2-6 of the session. Performance component means are based on all six trials.

Another interesting feature of the morphine results is that they do not have a clear counterpart in studies of nonspatial repeated acquisition, which have generally found $\mu$-opiate agonists to produce nonselective effects (Moerschbaecher \& Thompson, 1983; Moerschbaecher et al., 1985; but see also Schulze \& Paule, 1991) in monkeys. One possible explanation of the current results is that rats may be more sensitive than monkeys to the effects of morphine on acquisition. Although the behavioral effects of $\mu$-agonists are generally fairly consistent across species, species differences in complex discriminative performances with rats showing more sensitivity have been observed (e.g., Moerschbaecher, Mastropaolo, Winsauer, \& Thompson, 1984). However, a study of incremental repeated acquisition of response sequences in rats under morphine did not find evidence of selective disruption of acquisition (Paule \& McMillan, 1984).

Another interpretation of the current results is that the selective effects of morphine may depend on the type of task under study. The MST differs from more conventional operant procedures in many potentially relevant respects. For example, the effects of morphine on thermoregulation might be of special significance in this procedure; indeed, there is evidence that hypothermia can produce impairment in swim task performance (Rauch, Welch, \& Gallego,
1989). However, the selective nature of the morphine effects observed in the current study complicates a hypothermia interpretation because core temperatures would be equally affected in the performance and acquisition components (although it is possible that hypothermia might selectively impair acquisition). The spatial navigation requirement of the swim task is another obvious difference between the current research and most previous studies of repeated acquisition. It has been argued that spatial navigation involves unique neurobiological systems (Morris et al., 1986; O'Keefe \& Nadel, 1978); thus, drug effects might differ depending on the nature of the response requirement. More direct comparisons of drug effects on spatial and nonspatial learning tasks would be of considerable interest.

The $1.0 \mathrm{mg} / \mathrm{kg}$ dose of naloxone completely reversed the effects of morphine. This antagonism is consistent with an interpretation that morphine produced its effects in this study by means of an opiate receptor mechanism. None of the naloxone doses affected swimming in either acquisition or performance components when administered without morphine. The negative findings obtained with naloxone alone fail to replicate previous studies that showed facilitation of spatial learning by naloxone (Canli, Cook, \& Miczek, 1990; Decker, Introini-Collison, \& McGaugh, 1989; 

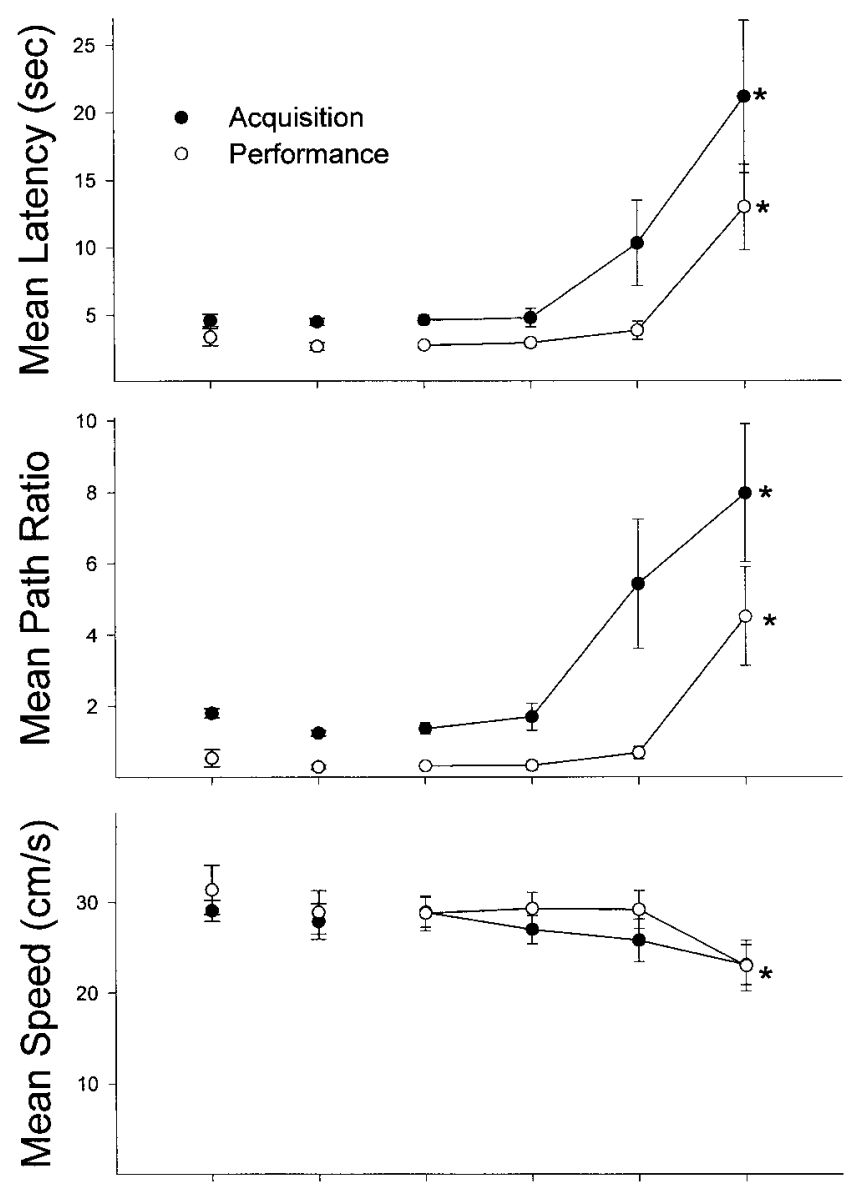

\section{BL Saline $0.3 \quad 1.0 \quad 3.0 \quad 5.6$ PCP Dose $(\mathrm{mg} / \mathrm{kg})$}

Figure 7. Mean escape latencies (top), swim path ratios (middle), and swim speeds (bottom) as a function of phencyclidine (PCP) dose during acquisition and performance components. Means for each subject were computed for all determinations of each dose used to compute the group mean presented here. When error bars are not visible, they are smaller than the symbol used to represent the data point. Points that differ significantly from saline controls are indicated by asterisks $(p<.05)$. BL $=$ baseline, or means of sessions not preceded by an injection; $\mathrm{sec}=$ seconds.

Gallagher, King, \& Young, 1983). However, the rapid acquisition noted under control conditions in the current study (see Figure 4) suggests that a floor effect may have prevented detection of any variable that increased acquisition (but note other failures to replicate facilitation of spatial learning by opiate antagonists: McDaniel, Mundy, \& Tilson, 1990; McNamara \& Skelton, 1991).

The finding that the competitive NMDA antagonist LY235959 generally impaired acquisition only at high doses that also interfered with performance was consistent with previous research that used the same procedure with the noncompetitive NMDA antagonist dizocilpine (Keith \&
Galizio, 1997) and the work of Cain et al. (Cain et al., 2000; Saucier et al., 1996) after nonspatial pretraining with both competitive and noncompetitive NMDA antagonists. However, the effects of PCP were more complex. Although there was no evidence of a selective effect at any dose in 3 of the 6 rats, the other 3 rats showed impairments in acquisition, but not performance, at some dose. The factors responsible for the individual differences in reactions to PCP are unclear; however, that PCP was more likely to be associated with selective disruption of acquisition than LY235959 is consistent with numerous other studies that show differences between PCP-like compounds and competitive NMDA antagonists in the tendency to produce a variety of adverse effects (see Wiley, 1997; Willetts et al., 1990). The current findings with PCP on repeated spatial acquisition showed rather less selectivity than has been noted in monkeys with nonspatial tasks (France et al., 1991; Moerschbaecher et al., 1985), but they support Cory-Slechta's (1994) observation that rats may be less sensitive in this regard. However, it should be recalled that Baron and Moerschbaecher (1996) found selective effects of dizocilpine and a competitive NMDA antagonist, CGS19755, in rats. Their procedure required between-group comparisons of performance and acquisition, and they used a food-reinforced, nonspatial task. Either of these procedural differences might explain the Baron and Moerschbaecher outcomes and those of Keith and Galizio (1997) and the current study. Evaluation of additional competitive and noncompetitive NMDA antagonists with the current procedure might clarify these issues.

In conclusion, we further demonstrated the utility of the multiple-component repeated-acquisition adaptation of the swimming pool navigation task as a baseline for experimental psychopharmacology. The procedure presented here may be more practical for many laboratories because, unlike the twopool procedure used by Keith and Galizio (1997), both components were performed in a single pool and two different stimulus patterns were used to distinguish the acquisition and performance components. As noted, relatively few multiplecomponent repeated-acquisition studies have used rats as subjects. Many studies of repeated acquisition in rats have adopted a simplified procedure (e.g., incremental repeated acquisition) that does not include an explicit performance component (Cohn \& Cory-Slechta, 1992; Paule \& McMillan, 1984; Winsauer, Bixler, \& Mele, 1996) or that involves between-group comparison of acquisition and performance (Baron \& Moerschbaecher, 1996). Our findings with PCP emphasize the importance of within-subject analyses that involve obtaining full dose-response functions for each subject. Similarly, other studies containing individual subject data analyses also have reported variation across subjects in the degree to which NMDA antagonists produce acquisition-selective effects (e.g., Thompson et al., 1987). Despite the value of the multiplecomponent procedure, the performance component is often omitted possibly because establishing stable discriminative control of multiple-response sequences in rats requires a great deal of training (e.g., a mean of 110 sessions in Winsauer, Rodriguez, Cha, \& Moerschbaecher, 1999). In the current study, stable acquisition and performance baselines were achieved by most rats in fewer than 30 training sessions $(M=$ 


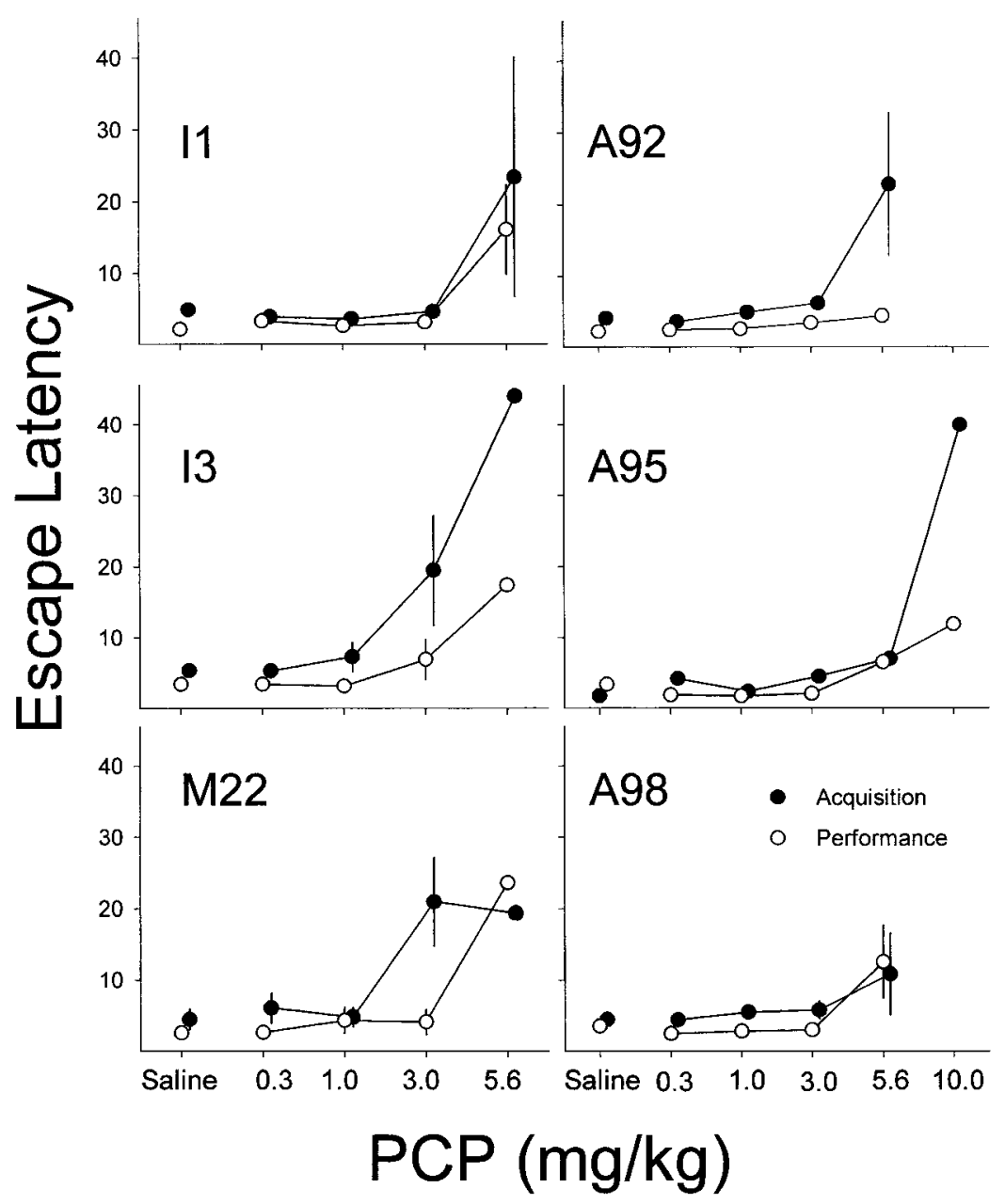

Figure 8. Individual escape latencies are presented as a function of phencyclidine (PCP) dose during acquisition and performance. Each point represents the means of all determinations of each dose, and vertical lines indicate standard error. Acquisition component means are based on Trials 2-6 of the session. Performance component means are based on all six trials.

28). Thus, the swimming pool navigation task may provide a useful tool for the study of repeated acquisition.

\section{References}

Allen, R. M., \& Dykstra, L. A. (2000a). Attenuation of mu-opioid tolerance and cross-tolerance by the competitive n-methyl-Daspartate receptor antagonist LY235959 is related to tolerance and cross-tolerance magnitude. Journal of Pharmacology and Experimental Therapeutics, 295, 1012-1021.

Allen, R. M., \& Dykstra, L. A. (2000b). Role of morphine maintenance dose in the development of tolerance and its attenuation by an NMDA receptor antagonist. Psychopharmacology, 148, 59-65.

Baron, S. P., \& Moerschbaecher, J. M. (1996). Disruption of learning by excitatory amino acid receptor antagonists. Behavioural Pharmacology, 6, 573-584.

Bisaga, A., Comer, S. D., Ward, A. S., Popik, P., Kleber, H. D., \& Fischman, M. W. (2001). The NMDA antagonist memantine attenuates the expression of opioid physical dependence in humans. Psychopharmacology, 157, 1-10.
Brooks, A. I., Cory-Slechta, D. A., Murg, S. L., \& Fedoroff, H. J. (2000). Repeated acquisition and performance chamber for mice: A paradigm for assessment of spatial learning and memory. Neurobiology of Learning and Memory, 74, 241-258.

Cain, D. P., Ighanian, K., \& Boon, F. (2000). Individual and combined manipulation of muscarinic, NMDA and benzodiazepine receptor activity in the water maze task: Implications for a rat model of Alzheimer dementia. Behavioral Brain Research, 111, 125-137.

Cain, D. P., Saucier, D., \& Boon, F. (1997). Testing hypotheses of spatial learning: The role of NMDA receptors and NMDA mediated long-term potentiation. Behavioral Brain Research, 84, 179-193.

Canli, T., Cook, R. G., \& Miczek, K. A. (1990). Opiate antagonists enhance the working memory of rats in the radial maze. Pharmacology Biochemistry and Behavior, 36, 521-555.

Cohn, J., \& Cory-Slechta, D. A. (1992). Differential effects of MK-801, NMDA and scopolamine on rats learning a fourmember repeated acquisition paradigm. Behavioral Pharmacology, 3, 403-413. 
Cohn, J., \& Cory-Slechta, D. A. (1993). Subsensitivity of leadexposed rats to the accuracy-impairing and rate-altering-effects of MK-801 on a multiple schedule of repeated learning and performance. Brain Research, 600, 208-218.

Cohn, J., \& Paule, M. G. (1995). Repeated acquisition of response sequences: The analysis of behavior in transition. Neuroscience and Biobehavioral Reviews, 19, 397-406.

Cory-Slechta, D. A. (1994). The impact of NMDA receptor antagonists on learning and memory functions. Psychopharmacology Bulletin, 30, 601-612.

Decker, M. W., Introini-Collison, I. B., \& McGaugh, J. L. (1989). Effects of naloxone on Morris water maze learning in the rat: Enhanced acquisition with pretraining but not posttraining administration. Psychobiology, 17, 270-275.

France, D. P., Moerschbaecher, J. M., \& Woods, J. H. (1991). MK801 and related compounds in monkeys: Discriminative stimulus effects and effects on a conditional discrimination. Journal of Pharmacology and Experimental Therapeutics, 257, 727-734.

Gallagher, M., King, R. A., \& Young, N. B. (1983). Opiate antagonists improve spatial memory. Science, 221, 1973-1978.

Heale, V., \& Harley, C. (1990). MK-801 and AP5 impair acquisition, but not retention, of the Morris milk maze. Pharmacology Biochemistry and Behavior, 36, 145-149.

Keith, J. R., \& Galizio, M. (1997). Acquisition in the Morris swim task is impaired by a benzodiazepine but not an NMDA antagonist: A new procedure for distinguishing acquisition and performance effects. Psychobiology, 25, 217-228.

McDaniel, K. L., Mundy, W. R., \& Tilson, H. A. (1990). Microinjection of dynorphin into the hippocampus impairs spatial learning in rats. Pharmacology Biochemistry and Behavior, 35, 429-435.

McNamara, R. K., \& Skelton, R. W. (1991). Pretraining morphine impairs acquisition and performance in the Morris water maze: Motivation reduction rather than amnesia. Psychobiology, 19, 313-322.

McNamara, R. K., \& Skelton, R. W. (1992). Pharmacological dissociation between the spatial learning deficits produced by morphine and diazepam. Psychopharmacology, 108, 147-152.

McNamara, R. K., \& Skelton, R. W. (1993). The neuropharmacological and neurochemical basis of place learning in the Morris water maze. Brain Research Reviews, 18, 33-49.

Moerschbaecher, J. M., Mastropaolo, J., Winsauer, P. J., \& Thompson, D. M. (1984). Effects of opioids on accuracy of a fixed-ratio discrimination in monkeys and rats. Journal of Pharmacology and Experimental Therapeutics, 230, 541-549.

Moerschbaecher, J. M., \& Thompson, D. M. (1980). Effects of phencyclidine, pentobarbital, and d-amphetamine on the acquisition and performance of conditional discriminations in monkeys. Pharmacology Biochemistry and Behavior, 13, 887-894.

Moerschbaecher, J. M., \& Thompson, D. M. (1983). Differential effects of prototype opioid agonists on the acquisition of conditional discriminations in monkeys. Journal of Pharmacology and Experimental Therapeutics, 226, 738-748.

Moerschbaecher, J. M., Thompson, D. M., \& Winsauer, P. J. (1985). Effects of opioids and phencyclidine in combination with naltrexone on the acquisition and performance of response sequences in monkeys. Pharmacology Biochemistry and Behavior, 22, 1061-1069.

Morris, R. G. M. (1981). Spatial localization does not require the presence of local cues. Learning and Motivation, 12, 239-260.

Morris, R. G. M., Anderson, E., Lynch, G. S., \& Baudry, M. (1986). Selective impairment of learning and blockade of long- term potentiation by an $N$-methyl-D-aspartate receptor antagonist, AP5. Nature, 319, 774-776.

O'Keefe, J., \& Nadel, L. (1978). The hippocampus as a cognitive map. Oxford, England: Oxford University Press.

Parsons, C. G., Danysz, W., \& Quack, G. (1999). Memantine is a clinically well tolerated $N$-methyl-D-aspartate (NMDA) receptor antagonist-A review of preclinical data. Neuropharmacology, 38, 735-767.

Paule, M. G., \& McMillan, D. E. (1984). Incremental repeated acquisition in the rat: Acute effects of drugs. Pharmacology Biochemistry and Behavior, 21, 431-439.

Peele, D. B., \& Baron, S. P. (1988). Effects of scopolamine on repeated acquisition of radial arm maze performance by rats. Journal of the Experimental Analysis of Behavior, 49, 275-290.

Perone, M. (1991). Experimental design in the analysis of freeoperant behavior. In I. H. Iverson \& K. A. Lattal (Eds.), Experimental analysis of behavior: Part I (pp. 135-172). Amsterdam: Elsevier.

Rauch, T. M., Welch, D. I., \& Gallego, L. (1989). Hypothermia impairs performance in the Morris water maze. Physiology and Behavior, 45, 315-320.

Robinson, G. S., Crooks, G. B., Shinkman, P. G., \& Gallagher, M. (1989). Behavioral effects of MK-801 mimic deficits associated with hippocampal damage. Psychobiology, 17, 156-164.

Saucier, D., Hargreaves, E. L., Boon, F., Vanderwolf, C. H., \& Cain, D. P. (1996). Detailed behavioral analysis of water maze acquisition under systemic NMDA or muscarinic antagonism: Nonspatial pretraining eliminates spatial learning deficits. $B e$ havioral Neuroscience, 110, 103-116.

Schulze, G. E., \& Paule, M. G. (1991). Effects of morphine sulfate on operant behavior in rhesus monkeys. Pharmacology Biochemistry and Behavior, 38, 77-83.

Thompson, D. M., \& Moerschbaecher, J. M. (1979). Drug effects on repeated acquisition. In T. Thompson \& P. B. Dews (Eds.), Advances in behavioral pharmacology Vol. 2, (pp. 229-259). New York: Academic Press.

Thompson, D. M., Winsauer, P. J., \& Mastropaolo, J. (1987). Effects of phencyclidine, ketamine and MDMA on complex operant behavior in monkeys. Pharmacology Biochemistry and Behavior, 26, 401-405.

Whishaw, I. Q. (1985). Formation of a place learning-set by the rat: A new paradigm for neurobehavioral studies. Physiology and Behavior, 35, 139-143.

Wiley, J. L. (1997). Behavioral pharmacology of N-methyl-Daspartate antagonists: Implications for the study and pharmacotherapy of anxiety and schizophrenia. Experimental and Clinical Psychopharmacology, 5, 365-374.

Willetts, J., Balster, R. L., \& Leander, J. D. (1990). The behavioral pharmacology of NMDA receptor antagonists. Trends in Pharmacological Science, 11, 423-428.

Winsauer, P. J., Bixler, M. A., \& Mele, P. C. (1996). Comparison of the effects of typical and atypical anxiolytics on learning in monkeys and rats. Journal of Pharmacology and Experimental Therapeutics, 276, 1111-1127.

Winsauer, P. J., Rodriguez, F. H., Cha, A. E., \& Moerschbaecher, J. M. (1999). Full and partial 5-HT-1A receptor agonists disrupt learning and performance in rats. Journal of Pharmacology and Experimental Therapeutics, 288, 335-347.

Received November 7, 2001 Revision received April 10, 2002 Accepted April 16, 2002 